\title{
Health Literacy and Self-Care of Patients with Heart Failure
}

\author{
Aleda M. H. Chen, Pharm.D., M.S.[Graduate Student], \\ Department of Pharmacy Practice, Center on Aging and the Life Course, Purdue University, West \\ Lafayette, Indiana
}

Karen S. Yehle, Ph.D., M.S., R.N.[Assistant Professor], School of Nursing, Purdue University, West Lafayette, IN

Kimberly S. Plake, Ph.D., R.Ph.[Associate Professor], School of Pharmacy, Purdue University, West Lafayette, IN

Matthew M. Murawski, Ph.D., R.Ph.[Associate Professor], and School of Pharmacy, Purdue University, West Lafayette, IN

Holly L. Mason, Ph.D.[Senior Associate Dean]

College of Pharmacy, Nursing, and Health Sciences, Purdue University, West Lafayette, IN

\begin{abstract}
Background and Research Objective-Today's complex health care system relies heavily on sophisticated self-care regimens. To navigate the system and follow self-care protocols, patients must be able to understand and use health information, which requires health literacy. However, nearly 90 million Americans lack the necessary health literacy skills to adequately care for themselves in the face of a complex healthcare system and self-care regimens. Understanding how to effectively care for one's self is thought to improve heart failure symptoms and patient outcomes, but little is actually known about how health literacy influences self-care in patients with heart failure. The purpose of this pilot study was to examine the relationship between health literacy and self-care of patients with heart failure.
\end{abstract}

Subjects and Methods-Patients with a diagnosis of heart failure were recruited from a variety of community settings. Participants completed the Short-Form Test of Functional Health Literacy (measured health literacy), the Self-Care Index of Heart Failure (measured self-care maintenance, management, and confidence), and a demographic questionnaire. Spearman's Rho correlations were used to assess the strength of the relationship between health literacy level and self-care scores.

Results and Conclusions-Among the 49 participants recruited, health literacy was positively related to self-care maintenance $\left(\mathrm{R}_{\mathrm{s}}=0.357, \mathrm{p}=0.006\right)$. Health literacy had a negative relationship with self-care management $\left(\mathrm{R}_{\mathrm{s}}=-0.573, \mathrm{p}=0.001\right)$. There was no association between health literacy and self-care confidence $\left(\mathrm{R}_{\mathrm{s}}=0.201, \mathrm{p}=0.083\right)$. This project provides preliminary data regarding the association between health literacy and self-care in heart failure, showing support for higher health literate patients performing more self-care maintenance, which has been shown to improve patient outcomes in heart failure. Patients with higher health literacy trended toward having greater self-care confidence, which can increase the likelihood of performing self-care, but

Corresponding Author: Aleda M. H. Chen, Pharm.D., M.S., amhess@ purdue.edu Phone: (419) 260-1478, Fax: (765) 496-1886. Publisher's Disclaimer: This is a PDF file of an unedited manuscript that has been accepted for publication. As a service to our customers we are providing this early version of the manuscript. The manuscript will undergo copyediting, typesetting, and review of the resulting proof before it is published in its final citable form. Please note that during the production process errors may be discovered which could affect the content, and all legal disclaimers that apply to the journal pertain. 
this finding was not significant. It was unexpected to find that lower health literate patients performed more self-care management.

\section{Keywords}

health literacy; self-care; heart failure

\section{Background}

Heart failure is a progressive chronic condition characterized by an impairment of the ventricles to fill or eject blood, leading to dypsnea, fluid retention, and eventually death. 1 Approximately 5.8 million Americans have a diagnosis of heart failure, with 670,000 new cases occurring per year. 2 Heart failure predominately affects individuals over the age of 65 and leads to more hospitalizations for older adults than any other health condition. 3 The number of older adults hospitalized with heart failure has increased significantly from 1980 to 2006.4 Estimates of the 2010 direct costs of heart failure are $\$ 39.2$ billion, with over 1.1 million hospitalizations and nearly 3.4 million ambulatory care visits contributing to costs. 2 Patients with heart failure should perform health-related activities, known as self-care, to improve outcomes in heart failure. $5^{-7}$

Self-care is a decision-making process whereby individuals choose to perform health behaviors that promote physiological stability (known as self-care maintenance) and respond to symptoms (known as self-care management).5 Self-care maintenance activities for heart failure patients include: monitoring weight daily, monitoring sodium and fluid intake, and exercising daily. Self-care management activities may include the following decisions: consulting a healthcare professional, reducing fluid and sodium intake, and increasing the dose of a diuretic.5 Self-care utilizes both the skills and knowledge of individuals.28, 29 Individuals with heart failure face significant challenges as they must not only gain knowledge specific to heart failure, but also apply this knowledge to specific situations, such as shortness of breath or fluid retention, that may arise.6 Self-care can be more challenging when there are barriers to gaining knowledge, such as inadequate health literacy.10

Health literacy is defined by the Institute of Medicine (2004) as "the degree to which individuals have the capacity to obtain, process, and understand basic health information and services needed to make appropriate health decisions." 10 Health literacy results from the interaction between individuals and the healthcare system, such as the experiences with healthcare providers and health-related materials that occur over an individual's life course. These interactions require a multitude of skills, including reading, writing, mathematics, speech, and comprehension. Examples of activities that require health literacy include interpreting prescription label directions, following directions before a medical procedure, and completing insurance paperwork. An estimated 12 percent of adults in the United States (U.S.) have the level of health literacy required to manage their health care, 11 and as many as 27 to 54 percent of patients with heart failure have low health literacy.12-16 The average individual in the U.S. reads at the eighth grade level, and Medicaid enrollees typically read at the fifth grade level.26, 27 However, the majority of health information is written at the tenth grade level or above.10, 26, 27

Inadequate health literacy can cause difficulties in understanding and following directions given within the healthcare system.15 For example, individuals with poorer health literacy have difficulty interpreting medication warning labels, 17 following directions on a prescription label,18 and identifying their medications.19 Difficulties in understanding and performing health-related activities may ultimately lead to poorer health outcomes.10, 15 Inadequate health literacy is associated with poorer physical and mental health,20 less 
healthcare knowledge, 21 poor medication adherence, 22 decreased use of preventive services, 21,23 increased hospitalizations, 24 and increased healthcare costs. 10 Individuals with lower health literacy also present with more advanced diseases20,24 and have a greater risk of all-cause mortality as well as cardiovascular-associated mortality.25 According to Evangelista and colleagues (2010), research is needed regarding further understanding of the relationship between health literacy and heart failure self-care in order to effectively deliver healthcare information to patients who have inadequate health literacy.15

If patients have inadequate health literacy and have not gained enough information to fully understand self-care, they may lack the belief, or self-confidence, in their ability to perform self-care. Evaluating and acting upon symptoms requires not only knowledge but the confidence to perform self-care behaviors. Without confidence, individuals are less likely to adhere to self-care.9, 30 Individuals with inadequate health literacy and heart failure may not be able to understand instructions provided or may lack confidence in self-care abilities, leading to poorer adherence to self-care.

The purpose of this pilot study was to examine the relationship between patient health literacy level and self-care maintenance, management, and confidence in patients with heart failure.

\section{Methods}

\section{Subjects and Procedures}

Participants were recruited using a non-probability convenience sample from a variety of Midwestern community settings, including two heart failure clinics (one urban and one rural), two continuing-care retirement communities (independent living residents only), a community hospital, a cardiology practice, and referrals from other participants. At both heart failure clinics, patients participated in a structured educational program over the first few months, and additional education was provided after program completion when necessary. Education at both clinics was directed by nurses, but includes a variety of other healthcare professionals as needed (e.g. dieticians). Participants recruited from settings other than heart failure clinics received a variety of education regarding their heart failure and the exact nature of this education was not known. Multiple settings were chosen in an attempt to obtain a diverse and more representative sample of community-dwelling patients with heart failure.

Information packets were distributed at research sites to potential participants. Packets contained an information sheet, a form for interested individuals to request additional information, and a business reply envelope addressed to the primary investigator. Interested individuals who were ages 18 years or older, had an established diagnosis of heart failure, could read and speak English, and were not cognitively impaired were contacted to participate in the study. Clinical judgment by nurses and researchers was used to determine the presence of cognitive impairment. Participants completed three questionnaires in person: the Short-Form Test of Functional Health Literacy in Adults (S-TOFHLA), the Self-Care Index of Heart Failure version 4 (SCHFI v.4), and a demographic questionnaire. Institutional Review Board (IRB) approval was obtained prior to data collection. Depending on the site and IRB requirements, verbal or written informed consent was obtained for each participant. Recruitment began in August 2008 and was completed in March 2010.

\section{Measurement Instruments}

The Short-Form Test of Functional Health Literacy in Adults (S-TOFHLA) was used to measure health literacy. This scale measures functional health literacy, which is the ability to use reading, writing, and computational skills at a level adequate to meet the needs of 
everyday life. The S-TOFHLA contains 36 questions that must be completed without assistance. Participants complete as many questions as possible in seven minutes. A total score is placed into one of three categories of health literacy: inadequate (scores 0 to 16), marginal (scores 17-22), and adequate (23-36).14 Individuals with inadequate health literacy scores misread the majority of directions and written information in the healthcare setting. Individuals with marginal scores perform better than those with inadequate skills, but still may have difficulty reading prescription labels. Adequate health literacy scores indicate that an individual can comprehend prescription labels and most written health information.10, 31

The S-TOFHLA is a reliable and valid measure of health literacy. The S-TOFHLA was validated utilizing 211 patients, in the same setting as the Test of Functional Health Literacy in Adults (TOFHLA, previously validated). Reliability of the S-TOFHLA was 0.98 for reading comprehension, as measured by Cronbach's alpha. The S-TOFHLA was also found to have goodness of fit, with $\mathrm{p}=0.50$ for inadequate health literacy cutoff and $\mathrm{p}=0.12$ for marginal health literacy cutoff.31 Criterion validity has been established with the full-length TOFHLA ( $\mathrm{r}=0.91)$ and the Rapid Estimation of Adult Literacy in Medicine ( $\mathrm{r}=0.80) .10,31$

The Self-Care Heart Failure Index version 4 (SCHFI v.4) was used to measure self-care. The SCHFI v.4 contains 15 items, with three self-care subscales: maintenance (Subscale A), management (Subscale B), and confidence (Subscale C). The maintenance subscale, which reflects treatment adherence, includes five items that measure behaviors related to the performance of exercise, daily weight monitoring, and diet adherence. Responses range from one (never) to four (always). The management subscale measures patients' decisionmaking process for implementing six treatments for heart failure exacerbation. Responses range from one (not likely) to four (very likely). The confidence subscale measures patients' self-confidence in their ability to perform self-care behaviors and make decisions related to heart failure, such as symptom evaluation, symptom changes, self-care actions, and the effectiveness of their actions. Responses range from one (not confident) to four (extremely confident). 5

Each subscale is scored from 0 to 100, with higher scores reflecting better self-care. A score of 70 or greater indicates adequate self-care. 8 Subscale B (self-care management) is scored only when participants have had symptoms in the past few months.5 Reliability for each of the SCHFI v. 4 subscales was as follows: 0.56 for maintenance (Subscale A), 0.70 for management (Subscale B), and 0.82 for confidence, as measured by Cronbach's alpha. While the internal consistency for the maintenance subscale (Subscale A) was lower than expected, it has been suggested that this may be due to health behaviors being independent and having multiple influential factors.5 Additionally, a longitudinal study found the alpha of the combined maintenance and management scales to be 0.798 .32

Demographic information was obtained, including gender, age, marital status, co-habitation, social support, quality of support, ethnicity/race, years of education, highest educational degree obtained, employment status, income, smoking history, alcohol usage, exercise recommendation, time spent exercising, height, weight, insurance, number of prescription medications, and place of residence (i.e. at home, retirement community, assisted living, or other).

\section{Data Analysis}

Statistical analyses were conducted using SPSS software for Windows (version 17.0; SPSS Inc, Chicago, Illinois). An a priori value of alpha $=0.05$ was set for statistical significance. Health literacy scores were categorized according to the S-TOFHLA cut-offs for inadequate, marginal, and adequate health literacy. Each SCHFI subscale was scored separately. Scoring 
procedures for the S-TOFHLA and the SCHFI v. 4 were followed as outlined by the authors of each scale.5, 31

Frequencies and percentages were calculated for categorical variables, including health literacy category. Means and standard deviations were calculated for continuous variables, including self-care subscales. Spearman's Rho correlations were used to measure relationships between health literacy category and self-care maintenance, management, and confidence.

Chi-square analysis was conducted to identify differences between demographic variables and health literacy category. Two-sample t-tests were performed to compare demographic variables containing two categories (ex: gender) and each self-care subscale. Comparisons between demographic variables containing three or more categories (ex: recruitment site) and each self-care subscale were performed using One-Way Analysis of Variance (ANOVA). If a statistically significant difference was identified, multiple comparisons (Bonferroni corrections) were performed to identify which categories contained the significant difference.

\section{Results}

\section{Participant Characteristics}

In total, 49 patients with heart failure were recruited. Participants typically were white, over the age of 65, retired, living at home, and had at least a high school education. Most participants did not drink or smoke, were told to exercise and had exercised in the previous week, were overweight or obese, and had some type of health insurance coverage (Table 1).

\section{Health Literacy Characteristics and Demographic Relationships}

The highest percentage of participants had adequate health literacy $(71.4 \%)$, followed by inadequate (16.3\%), and marginal (12.2\%) (see Table 2). Higher educational status (high school or greater) was related to higher health literacy $(\mathrm{p}=0.007)$. Participants who reported being instructed to use a structured exercise program as well as those who reported exercising at least one hour in the last week were more likely to have higher health literacy ( $\mathrm{p}=0.015$ and $\mathrm{p}=0.019$, respectively).

\section{Self-Care Characteristics and Demographic Relationships}

Participants had a higher level of self-care maintenance (mean score $=76.74 \pm 20.98$ ) than confidence in self-care (mean score $68.60 \pm 22.31$ ) or in self-care management for the 26 participants with symptoms within the previous three months (mean score $=65.05 \pm 24.47$ ). Participants with at least a high school education had greater self-care confidence than participants who did not complete high school $(\mathrm{p}=0.002)$. No other statistically significant differences were found between the demographic variables and self-care subscales.

\section{Health Literacy and Self-Care Relationships}

Health literacy was positively related to self-care maintenance $(0.357, \mathrm{p}=0.006)$ and negatively related to self-care management $(-0.573, \mathrm{p}=0.001)$. There was no association between health literacy and self-care confidence $(0.201, \mathrm{p}=0.083)$.

\section{Discussion}

In this pilot study, there were relationships between health literacy and self-care, with evidence suggesting that higher health literacy was related to better self-care maintenance and lower health literacy was associated with better self-care management. In order for 
patients with heart failure to perform self-care, they must be able to understand and use the healthcare information provided.5, 6, 28, 29 If patients have inadequate health literacy, they are more likely to have difficulty in understanding health information and acting upon it to perform self-care.10 This has clinical relevance, as better self-care ability is associated with decreased hospitalizations, mortality, and costs.33 Although self-care was not specifically measured, educational interventions aimed at patients with inadequate health literacy also reduced all-cause mortality in heart failure.14, 34

Higher health literacy was related to the greater performance of self-care maintenance. This finding may have important implications in the provision of patient care. Tailored education according to health literacy level may be necessary to improve self-care maintenance behaviors in patients with heart failure. Future research should examine whether health literacy level-specific education improves self-care.

It was unexpected to find that lower levels of health literacy were related to greater adherence to self-care management behaviors. There is no evidence in the literature to confirm this finding. Since only half of the participants had symptoms within the past few months, a larger sample size may provide more information to confirm this finding. Furthermore, patients with heart failure may be more likely to take care of themselves when symptoms occur, as uncomfortable symptoms may prompt action. Daily maintenance activities may have less apparent health prompts, reducing an individual's likelihood to perform these activities. Individuals with lower health literacy also had poorer self-care maintenance, which may lead to an increased likelihood of experiencing symptoms and more opportunities to learn about managing symptoms. Future research should not only confirm the relationship between health literacy and self-care management but also explore the various reasons as to why this occurs.

Although not statistically significant, a positive trend between health literacy and selfconfidence was observed in this study. In diabetes, a positive relationship between confidence and health literacy was found.35 Since the time since diagnosis was not controlled for in this study, participants recruited have managed their heart failure symptoms for different lengths of time. Prior success in maintaining health and managing symptoms can increase confidence, 9,30 and individuals may be confident in their skills due to past successes, regardless of health literacy level. Support from family, friends, and other key individuals also may contribute to self-care confidence. Adequate support improves confidence, 9,30 and individuals of all health literacy levels may have varying levels of support. Most individuals $(n=44)$ in this study indicated that they received good or very good support from others, support level is an unlikely explanation for the lack of a relationship between health literacy and self-care confidence. Further testing with a larger sample size is needed to determine if heart failure self-care confidence and health literacy are related, while controlling for length of time since diagnosis and support.

In this study, self-care maintenance in participants was considered adequate $(\mu=76.74)$, as the cut-off for adequacy is a score of 70 or greater. 8 Self-care management and self-care confidence scores did not reach the minimum score for adequacy, indicating that the overall management skills and confidence in self-care were not sufficient in this sample.

There are several limitations to this study. The sample size was small, with unequal numbers of participants with adequate, marginal, and inadequate health literacy. With a larger sample size, there could have been further examination of differences in health literacy and self-care scores. Despite this limitation, a statistically significant relationship was found between health literacy and self-care maintenance and management. Additional statistical analyses, such as linear regression, were unable to be performed in this pilot study. Linear regression 
analyses could have controlled for other factors associated with health literacy, such as educational attainment.10 These factors may explain some of the association between health literacy and self-care and future research should be powered to examine this. Prior education regarding heart failure was not controlled for in this study, as participants were recruited from diverse settings with varying length of time since diagnosis. However, the diversity of settings was intentional, as this was a naturalistic study. Although multiple settings were used in an attempt to recruit a more representative sample, participants tended to be white, higher income, and have adequate health literacy. This study is a cross-sectional design and provides information regarding health literacy and self-care at a single point in time.

Longitudinal studies are needed to examine the relationship between health literacy and selfcare over time to understand how health literacy interacts within the self-care process in heart failure.

\section{Conclusion}

Health literacy was positively related to self-care maintenance, negatively related to selfcare management, and not related to confidence in ability to perform self-care. These results raise a new awareness of the negative association of health literacy and self care management that requires further research and provide an impetus for development and testing of health literacy-level specific educational interventions to improve self-care in patients with heart failure.

\section{Acknowledgments}

This research project was supported, in part, by a grant from the Regenstrief Center for Healthcare Engineering at Purdue University. Support for Aleda M. H. Chen was provided by the National Institute on Aging (T32AG025671) and the Purdue University Center on Aging and the Life Course.

\section{References}

1. Lindenfeld J, Albert NM, Boehmer JP, et al. HFSA 2010 comprehensive heart failure practice guideline. Journal of Cardiac Failure. 2010; 16(6):e1-e194. [PubMed: 20610207]

2. Lloyd-Jones D, Adams RJ, Brown TM, et al. Heart disease and stroke statistics--2010 update: A report from the American Heart Association. Circulation. 2010; 121(7):e46-e215. [PubMed: 20019324]

3. Ni H, Nauman D, Burgess D, Wise K, Crispell K, Hershberger RE. Factors influencing knowledge of and adherence to self-care among patients with heart failure. Archives of Internal Medicine. 1999; 159(14):1613-1619. [PubMed: 10421285]

4. Liu L. Changes in cardiovascular hospitalization and comorbidity of heart failure in the United States: Findings from the National Hospital Discharge Surveys 1980-2006. International Journal of Cardiology. 2010 In Press, Corrected Proof.

5. Riegel B, Carlson B, Moser DK, Sebern M, Hicks FD, Roland V. Psychometric testing of the SelfCare of Heart Failure Index. Journal of Cardiac Failure. 2004; 10(4):350-360. [PubMed: 15309704]

6. Artinian NT, Magnan M, Sloan M, Lange MP. Self-care behaviors among patients with heart failure. Heart \& Lung: The Journal of Acute and Critical Care. 2002; 31(3):161-172.

7. Rockwell JM, Riegel B. Predictors of self-care in persons with heart failure. Heart \& Lung: The Journal of Acute and Critical Care. 2001; 30(1):18-25.

8. Riegel B, Lee CS, Dickson VV, Carlson B. An update on the Self-Care of Heart Failure Index. Journal of Cardiovascular Nursing. 2009; 24(6):485-497. [PubMed: 19786884]

9. Bandura A. Self efficacy: Toward a unifying theory of behavioral change. Psychological Review. 1977; 84(2):191-215. [PubMed: 847061]

10. Institute of Medicine. Health Literacy: A Prescription to End Confusion. National Academy of Sciences; 2004. 
11. Agency for Healthcare Research and Quality. National healthcare disparities 2007 report. 2008. p. $1-252$.

12. Laramee AS. Relationship of literacy and heart failure in adults with diabetes. BMC Health Services Research. 2007; 7

13. Morrow D, Wu J, Murray MD, et al. Correlates of health literacy in patients with chronic heart failure. The Gerontologist. 2006; 46(5):669-676. [PubMed: 17050758]

14. DeWalt DA, Corr KE, Kosner MC, et al. A heart failure self-management program for patients of all literacy levels: A randomized, controlled trial. BMC Health Services Research. 2006; 6:30-40. [PubMed: 16533388]

15. Evangelista LS, Rasmusson KD, Laramee AS, et al. Health literacy and the patient with heart failure - Implications for patient care and research: A consensus statement of the Heart Failure Society of America. Journal of Cardiac Failure. 2010; 16(1):9-16. [PubMed: 20123313]

16. Gazmararian JA, Baker DW, Williams MV, et al. Health literacy among Medicare enrollees in a managed care organization. JAMA: Journal of the American Medical Association. 1999; 281(6): 545-551.

17. Davis TC, Wolf MS, Bass PF III, et al. Low literacy impairs comprehension of prescription drug warning labels. JGIM: Journal of General Internal Medicine. 2006; 21(8):847-851.

18. Davis TC, Wolf MS, Bass PF III, et al. Literacy and misunderstanding prescription labels. Annals of Internal Medicine. 2006; 145:887-894. [PubMed: 17135578]

19. Kripalani S, Henderson LE, Chiu EY, Robertson R, Koim P, Jacobson TA. Predictors of medication self-management skill in a low-literacy population. JGIM: Journal of General Internal Medicine. 2006; 21(8):852-856.

20. Wolf MS, Gazmararian JA, Baker DW. Health literacy and functional health status among older adults. Archives of Internal Medicine. 2005; 165(17):1946-1952. [PubMed: 16186463]

21. DeWalt DA, Berkman ND, Sheridan S, Lohr KN, Pignone MP. Literacy and health outcomes. JGIM: Journal of General Internal Medicine. 2004; 19(12):1228-1239.

22. Gazmararian JA, Kripalani S, Miller MJ, Echt KV, Junling R, Rask K. Factors associated with medication refill adherence in cardiovascular-related diseases. JGIM: Journal of General Internal Medicine. 2006; 21(12):1215-1221.

23. Scott TLP, Gazmararian JA, Williams MV, Baker DW. Health literacy and preventive health care use among Medicare enrollees in a managed care organization. Medical Care. 2002; 40(5):395404. [PubMed: 11961474]

24. Baker DW, Parker RM, Williams MV, Clark WS. Health literacy and the risk of hospital admission. JGIM: Journal of General Internal Medicine. 1998; 13(12):791-798.

25. Baker DW, Wolf MS, Feinglass J, Thompson JA, Gazmararian JA, Huang J. Health literacy and mortality among elderly persons. Archives of Internal Medicine. 2007; 167(14):1503-1509. [PubMed: 17646604]

26. Kirsch, IS.; Jungeblut, A.; Jenkins, L.; Kolstadt, A. Adult literacy in America. Washington, D.C.: National Center for Education Statistics, U.S. Department of Education; 1993.

27. Weiss BD, Coyne C. Communicating with patients who cannot read. New England Journal of Medicine. 1997; 337(4):272-274. [PubMed: 9227936]

28. Orem, D. Nursing: Concepts of Practice. 6th ed.. St. Louis: Mosby; 2001.

29. Orem, D. Self-care and health-promotion: understanding self-care. In: McLaughlin, K.; Taylor, SG., editors. Self-care theory in nursing: Selected papers of Dorothea Orem. New York, NY: Springer Publishing Co, Inc.; 2003.

30. Yehle KS, Plake KS. Self-efficacy and educational interventions in heart failure: A review of the literature. Journal of Cardiovascular Nursing. 2010; 25(3):175-188. [PubMed: 20386241]

31. Baker DW, Williams MV, Parker RM, Gazmararian JA, Nurss J. Development of a brief test to measure functional health literacy. Patient Education and Counseling. 1999; 38(1):33-42. [PubMed: 14528569]

32. Yehle, KS. A comparison of standard office visits and shared medical appointments in adults with heart failure [Dissertation]. Cypress, CA: Touro University International; 2007. 
33. Riegel B, Moser DK, Anker SD, et al. State of the science. Promoting self-care in persons with heart failure. A scientific statement from the American Heart Association. Circulation. 2009; 120(12):1141-1163. [PubMed: 19720935]

34. DeWalt DA, Pignone M, Malone R, et al. Development and pilot testing of a disease management program for low literacy patients with heart failure. Patient Education and Counseling. 2004; 55(1):78-86. [PubMed: 15476993]

35. Schillinger D, Grumbach K, Piette J, et al. Association of health literacy with diabetes outcomes. JAMA: Journal of the American Medical Association. 2002; 288(4):475. 
Table 1

Characteristics of Participants

\begin{tabular}{|l|r|}
\hline Socio-Demographic Information & $\begin{array}{r}\text { Participants } \\
\mathbf{4 9}(\boldsymbol{\%})\end{array}$ \\
\hline Mean age (years) \pm SD & $72 \pm 13.3$ \\
\hline Males & $33(67.3 \%)$ \\
\hline Married & $26(53.1 \%)$ \\
\hline Lives at home & $40(81.6 \%)$ \\
\hline Good or very good support from family and friends & $44(89.7 \%)$ \\
\hline White/caucasian & $45(91.8 \%)$ \\
\hline Completed year 12 education & $43(87.8 \%)$ \\
\hline Retired & $35(71.4 \%)$ \\
\hline At least enough income to make ends meet & $45(91.8 \%)$ \\
\hline Former smoker & $24(49.0 \%)$ \\
\hline One or less alcoholic beverage per week & $43(87.8 \%)$ \\
\hline Recommended to use a structured exercise program & $37(75.5 \%)$ \\
\hline Exercised at least one hour in past week & $26(53.1 \%)$ \\
\hline Obese BMI & $24(49.0 \%)$ \\
\hline Prescribed at least 8 prescriptions & $25(51.0 \%)$ \\
\hline Government-sponsored health insurance & $39(79.6 \%)$ \\
\hline Patient at urban or rural heart failure clinic & $32(65.3 \%)$ \\
\hline
\end{tabular}

BMI = Body Mass Index 
Table 2

Participant Health Literacy

\begin{tabular}{|l|r|}
\hline Health Literacy Category & $\mathbf{N}(\boldsymbol{\%})$ \\
\hline Adequate & $35(71.4 \%)$ \\
\hline Marginal & $6(12.2 \%)$ \\
\hline Inadequate & $8(16.3 \%)$ \\
\hline
\end{tabular}

